\title{
Aberrated Edge imaging with annular apertures
}

\author{
A. Narsaiah, M.Venkanna* and D. Karuna Sagar \\ Optics Research Group, Department of Physics, Osmania University Hyderabad, India-500007
}

\begin{abstract}
In this paper, we have studied the aberrated images of coherently illuminated straight edge objects with annular aperture in the presence of apodisation. The analytical studies were made for annular apertures apodised with Shaded aperture under the influence of defocus and primary spherical aberrations. The effect of aperture obscuration and apodisation on the edge ringing and peak intensity has been considered.
\end{abstract}

Key words: Aberrations, Annular aperture, Coherence, Edge objects, imaging systems.

\section{Introduction}

The studies concerning the images produced by optical systems with aberration-free and the improvement in the resulting images are almost completed in the limiting cases of coherent and incoherent illumination. Most of the past investigations [1-8] concerning the diffraction theory of partial coherence, where they confined to the idealized situations in which only aberration-free and uniformly illuminated systems were considered. However, the literature is rich in the studies on circular apertures, employing apodisation to suppress the fringing [9-16]. In the early cases K. SURENDER, P.K. MONDAL etc. have carried out studies on aberration free and non-uniform illuminated systems with annular aperture and redefined the edge-ringing [17].

Apodisation can be accomplished in several ways i.e., by altering the shape of the aperture or its transmission characteristics (HECHT and ZAJAC, 1987). The former is known as "Aperture Shaping" in which the shape of the aperture is altered from circular to non-circular. The later is known as "Aperture Shading" by using a spatial filter over the pupil from point to point (MONDAL and VENKAT REDDY, 1987). Thus apodisation is the process of changing the energy distribution in the point spread function by deliberate manipulation of the pupil function so as to improve some measure of the image quality (WETHERELL, 1980). In this paper we proposed Aperture shaping along with shaded aperture in the presence of defocus and primary spherical aberration. For this rotationally symmetric, diffraction-limited and coherent optical system has been considered.

It has been known for some time that, stop employing at the center of the aperture of an optical system i.e., using annular aperture-makes the central maximum in the airy pattern narrower and increases the depth of focus. Rayleigh pointed out the narrowing of the central maximum and the simultaneous increase in intensity in the outer diffraction rings, and Steward showed that the depth of focus, as judged by the intensity at the center of the out-of-focus airy pattern, is increased. In the previous paper, we proposed the effects of apodisation on the diffraction images of coherently illuminated straight edge using shrink aperture [18].

\section{Theory}

An opaque straight edge is one which is bright on one side of a line and dark on the other.

The mathematical representation of amplitude transmission of an opaque straight edge is given by

$$
\begin{array}{ll}
\mathrm{A}(\mathrm{u}, \mathrm{v})=1 & \text { for } \mathrm{u} \geq 0 \\
\mathrm{~A}(\mathrm{u}, \mathrm{v})=0 & \text { for } \mathrm{u}<0
\end{array}
$$

This indicates that the transmission function is discontinuous at $\mathrm{u}=0$. The Fourier transform for this equation gives the amplitude spectrum of the object and is given by [18]

$$
a(x, y)=\frac{1}{2} \cdot \delta(y)\left[\delta(x)+\frac{1}{i \pi x}\right]
$$

where $\delta(x)$ is the Dirac-delta function. The modified object amplitude spectrum at the exit pupil of the optical system is given by

$$
a^{\prime}(x, y)=a(x, y) \cdot f(x, y)
$$


where $f(x, y)$ is the pupil function of the optical system. For the given optical system the complex amplitude distribution in the image plane is given by the inverse Fourier transform of expression (3). Thus

$$
A\left(u^{\prime}, v^{\prime}\right)=\iint_{\text {pupil }} a(x, y) \cdot f(x, y)\left\{\exp 2 \pi i\left(u^{\prime} x+v^{\prime} y\right)\right\} d x d y
$$

The present work constitutes one-dimensional edge condition and hence, the general form of amplitude distribution is given by

$$
A^{\prime}\left(u^{\prime}, v^{\prime}\right)=\frac{1}{2}+\frac{1}{\pi} \int_{0}^{1} f(x, 0) \frac{\sin (Z x)}{x} d x
$$

where $Z=2 \pi u^{\prime}$ and $f(x, 0)$ is the coherent transfer function of the system. The coherent transfer function $f(x, 0)$ in the current study is rotationally symmetric and satisfies the condition

$$
f(x, 0)=f(-x, 0)
$$

Pupil function $\mathrm{f}(\mathrm{r})$ for the shaded aperture is given by

$$
f(r)=1-\beta r^{2}
$$

where $r$ is the normalized distance of an arbitrary point on the pupil from its centre and $\beta$ is the apodisation parameter. The term $\beta$ controls the degree of non-uniformity of transmission over the pupil. A value of $\beta=0$, corresponds to diffraction limited airy system having uniform transmission of unity over the entire aperture. On introducing wave aberrations such as defocus and primary spherical aberration expression (5) takes the form

$$
A^{\prime}\left(u^{\prime}, v^{\prime}\right)=\frac{1}{2}+\frac{1}{\pi} \int_{0}^{1} f(r) \exp \left(-i\left(\phi_{d} \frac{x^{2}}{2}+\phi_{s} \frac{x^{4}}{4}\right)\right) \frac{\sin (Z x)}{x} d x
$$

For the given annular aperture the expression (8) becomes

$$
A^{\prime}\left(u^{\prime}, v^{\prime}\right)=\frac{1}{2}+\frac{1}{\pi} \int_{\varepsilon}^{1}\left(1-\beta r^{2}\right) \exp \left[-i\left(\phi_{d} \frac{x^{2}}{2}+\phi_{s} \frac{x^{4}}{4}\right)\right] \frac{\sin (Z x)}{x} d x
$$

Where $0 \leq \varepsilon<1$ is the central obscuration parameter.

Now the intensity distribution of an edge image formed by an apodised optical system is given by the squared modulus of expression (9).

Thus

$$
\begin{aligned}
& B\left(u^{\prime}\right)=B(Z)=\left|A^{\prime}\left(u^{\prime}\right)\right|^{2} \\
& B(Z)=\left|A^{\prime}(Z)\right|^{2}=\left|\frac{1}{2}+\frac{1}{\pi} \int_{\varepsilon}^{1}\left(1-\beta r^{2}\right) \exp \left[-i\left(\phi_{d} \frac{x^{2}}{2}+\phi_{s} \frac{x^{4}}{4}\right)\right] \frac{\sin (Z x)}{x} d x\right|^{2}
\end{aligned}
$$

The investigations on the effects of aberrations and obscuration parameter on the images of edge objects formed by coherent optical systems apodised by the shaded aperture have been evaluated using the expressions (10) by employing Matlab7.8. The intensity distribution B (Z) in the images of straight edge objects has been obtained for different values of dimensionless diffraction variable $Z$ varying from -10 to +20 . The central obscuration parameter of the aperture considered are $\varepsilon=0,0.1,0.2,0.3$ and 0.4 . However the value $\varepsilon=0$ represents the circular aperture.

The edge ringing is the ratio of the maximum positive deviation of the edge image to the geometric value of unity [19]. In effect it is the difference between the first maximum intensity of the fringes and the uniform intensity of unity. However, for annular aperture, as the maximum intensity is less than unity, edge ringing is redefined as the value by which the maximum peak in the image is below unity. It is thus negative for the case of annular apertures. 


\section{Results And Interpretation}

From the pupil transmission curves, it is clear that for $\beta=0, f(r)=1$ which implies uniform transmittance over the exit pupil. For this apodizer the amplitude transmittance decreases monotonically from the center towards the edges of the pupil. As the pupil transmittance is decreased at the edges as compared to that of the center, due to apodization, the result is reduction in the higher spatial frequency components in the image. This manifests as partial or full suppression of the undesired optical side lobes or secondary maxima, which consequently enhances edge imaging features.

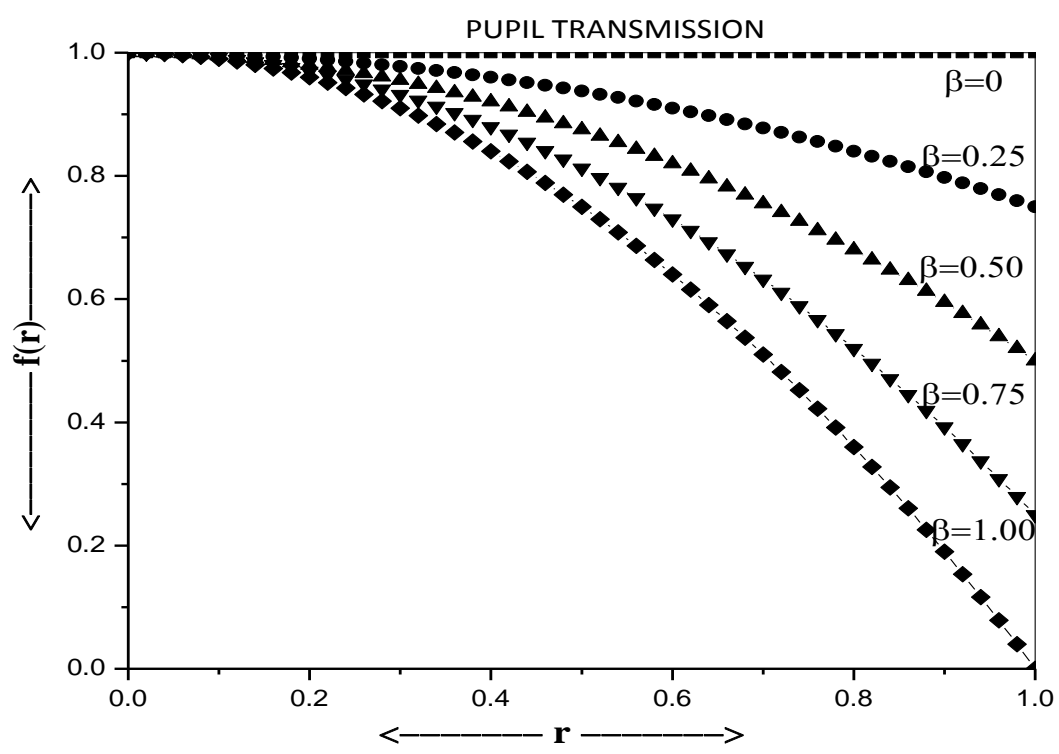

The intensity distribution of the straight edge for the airy case for both circular and annular apertures is given in fig.1. As the system is unapodised the edge ringing is pronounced for the circular aperture. For annular apertures, in general, the negative maximum amplitude increases with central obscuration $\varepsilon$ and hence the presence of ringing is much more pronounced. It is observed that for annular apertures the unwanted fringes of increasing intensity on the dark side of the edge. Since we considered the Fraunhoffer diffraction, there will be spurious fringes due to edge ringing on both the sides of the edge though the intensity is less on the dark side. On the bright side of the edge the max intensity falls as the annular zone is increasing but fringe intensity is in increasing trend on the dark side.

The fig. 2 gives the intensity distribution for the unapodised system in the presence of defocus and primary spherical aberrations for both circular and annular apertures. The same features have been observed from the fig. 2 except that the edge ringing is slightly reduced for the circular aperture even in the presence of aberrations.

Figs. 3 and 4 give the intensity distributions for the apodised annular apertures. A decrease in the peak intensities on the bright side is observed with apodisation. However, those on the dark side are almost unchanged. The edge ringing is increasing from $\varepsilon=0$ to 0.4 . All the minima on the dark side are non-zero for the annular aperture, which reduces the central-lobe intensity and increases the side-lobe intensity in the PSF response. Fig.5. shows the variation of the peak intensities with aperture obscuration $(\varepsilon)$ in the presence of aberrations for both the unapodised and apodised optical systems. The peak intensity falls by $50.13 \%$ for aberration free, unapodised optical systems and it is $64.13 \%$ for $\beta=1$ and $\phi_{s}=\phi_{d}=\pi$. Hence it is observed that the apodisation with the shaded aperture reduces the edge-ringing for both the circular and annular apertures. 


\section{IMAGE INTENSITY DISTRIBUTIONS}
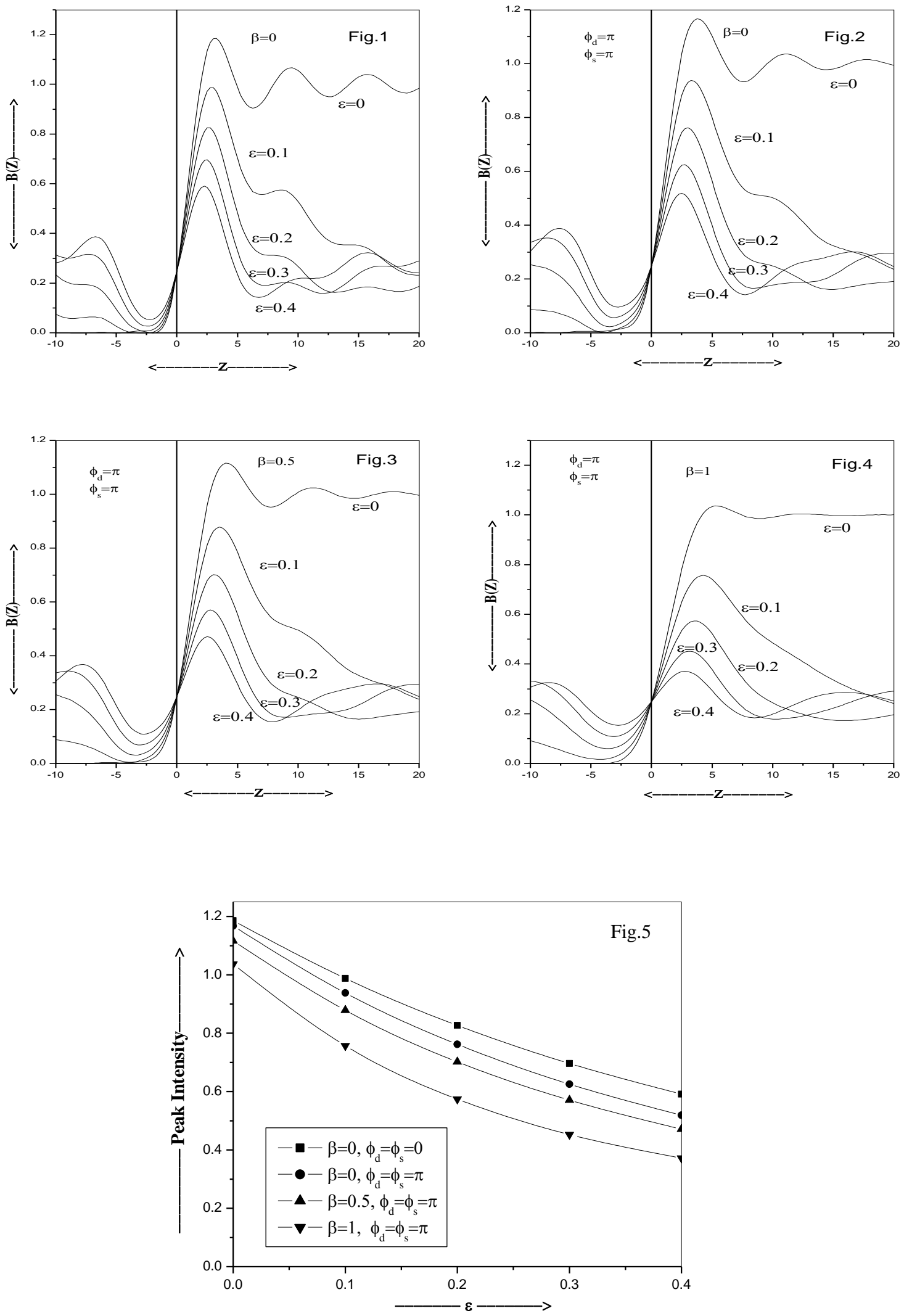


\section{Conclusions}

Central obscuration of the apertures aggravates the problem of the edge-ringing and there is a loss of edge gradient. Apodisation reduces the edge ringing for circular and annular apertures even in the presence of aberrations. However, this can be achieved only at the cost of increase of edge shifting and a loss of edge gradient. The intensity of spurious fringes produced in the dark side is relatively high with annular apertures and is in increasing trend with obscuration, while it is reverse in the bright side. Finally, it is observed that central aperture obscuration introduces an asymmetry in the image intensity distribution pattern and the definition of the edge image is totally lost.

\section{References}

[1]. G. B. Parrent, Jr. and T. J. Skinner, Opt. Acta 8, 93 (1961).

[2]. R. A. Shore, Proc. of the Symposium on ETA, Copenhagen, Denmark, p. 787 (Pergamon, London, 1963).

[3]. W. T. Cathey, J. Opt. Soc. Am. 55, 1035 (1965)

[4]. R. A. Shore, B. J. Thompson, and R. E. Whitney, J. Opt. Soc. Am. 56, 733 (1966)

[5]. J. G. Meadors, Opt. Acta 12, 379 (1965).

[6]. R. A. Shore, J. Opt. Soc. Am. 58, 1484 (1968).

[7]. K. Singh and H. S. Dhillon, J. Opt. Soc. Am. 59, 397 (1969).

[8]. K. Singh and B. N. Gupta, Opt. Acta 17, 609 (1970).

[9]. R. W. Smith, Opt.Commu.4, 157(1971).

[10]. R. W. Smith, Opt.Commu.6, 8(1972).

[11]. R. W. Smith, Opt.Commu.9, 61(1973).

[12]. F.G. Leaver and R. W. Smith, Optics 39,158(1973).

[13]. T.Araki and T. Asakura, Opt.Commun.20, 3, 373(1977).

[14]. K. P. Rao, P.K. Mondal and T.Sheshagiri Rao, Optik, 50, 73(1977)

[15]. J. P. Mills and B.J. Thampson, J. O. S. A(A), 3, 704(1985).

[16]. J. Sethuraman and R.S. Sirohi. Opt. Commun. 28, 1, 11(1979).

[17]. Surender K, Goud S L \& Mondal P K, J Opt, 21 (3), 75 (1992).

[18]. M.Venkanna and D.K.Sagar, IJIRSET, 2, 3, 645(2013).

[19]. T. Asakura and T. Araki, Optik, 46, 365(1976). 